\title{
Local solutions to positive characteristic non-Archimedean differential equations
}

\author{
João Pedro Pinto dos Santos
}

\begin{abstract}
In the complex domain, one can integrate (solve) holomorphic ordinary differential equations (ODEs) near a non-singular point. We study the existence of solutions in the case of a positive characteristic base field $k$ which is complete with respect to a non-Archimedean absolute value. ODEs are substituted by modules over a ring of analytic functions endowed with an action of all differential operators. The monodromy groups associated to the corresponding category are computed.
\end{abstract}

\section{Introduction}

Cauchy established the existence and uniqueness of local solutions to ordinary differential equations in the complex domain. Nowadays, the usual proof makes use of some contraction principle in complete metric spaces, but Cauchy's proof is, by far, the most interesting one for the algebraist. It consists of: (1) solving the equations formally in power series; and (2) taking care of convergence (see, for example, the last chapter of [Car61]). Once that has been done, the general theory of complex analysis assures: (3) convergence in bigger domains. In the characteristic zero non-Archimedean setting (3) fails but (1) and (2) can be carried [DGS94, III, 5]. The goal of this work is to understand (2) in the case of an algebraically closed base field $k$ of positive characteristic which is complete with respect to a non-trivial non-Archimedean absolute value $|\cdot|: k^{*} \rightarrow \mathbb{R}_{>0}$.

The geometric analogue of differential equations in positive characteristic are modules having the action of all differential operators. For these modules one is presented with the basic question of convergence of formal solutions. That is, let $R$ be a ring of power series $\sum_{0}^{\infty} a_{i} x^{i}$ with some boundedness condition on the $\left|a_{i}\right|$ (for example, that given in Definition 2 below). Let $\partial_{n}$ be the operators analogous to

$$
\frac{1}{n !} \frac{d^{n}}{d x^{n}}
$$

on $R$. A linear stratified differential equation of rank $\mu$ is a system of equations

$$
\begin{gathered}
\partial_{n} y_{1}=b_{11}^{(n)} y_{1}+\cdots+b_{1 \mu}^{(n)} y_{\mu} \\
\vdots \\
\partial_{n} y_{\mu}=b_{\mu 1}^{(n)} y_{1}+\cdots+b_{\mu \mu}^{(n)} y_{\mu}
\end{gathered} \quad(n \in \mathbb{N})
$$

where the $b_{i j}^{(n)}$ are in $R$ and the matrices $\left(b_{i j}^{(n)}\right)$ satisfy some set of relations. These relations reflect the relations between the operators $\partial_{n}$, such as $\left(\partial_{1}\right)^{p}=0$ and $\partial_{0}=$ id. If we were in characteristic zero,

Received 16 November 2006, accepted in final form 21 February 2007, published online 9 November 2007.

2000 Mathematics Subject Classification 12H05 (primary), 12F12, 14G22 (secondary).

Keywords: differential operators in positive characteristic, Tannakian fundamental groups, rigid geometry, differential Galois theory.

This work is part of the author's Cambridge $\mathrm{PhD}$ thesis supervised by N. I. Shepherd-Barron and financially supported by Trinity College.

This journal is (C) Foundation Compositio Mathematica 2007. 


\section{J. P. P. DOs SAntos}

the constraints would restrict the $\left(b_{i j}^{(n)}\right)$ in such a way that $\left(b_{i j}^{(1)}\right)$ is already sufficient information to determine the other matrices. It is quite easy to see that these equations will always have solutions $\left(y_{1}, \ldots, y_{\mu}\right)$ in the ring $k[[x]]$; in fact, there exists a $Y=\left(y_{i j}\right) \in G L_{\mu}(k[[x]])$ whose columns are solutions. Moreover, because the space of solutions will have $k$-dimension at most $\mu$, it follows that any other $\mu \times \mu$ invertible matrix whose columns are solutions to (1) is of the form $Y \cdot A, A \in G L_{\mu}(k)$.

We then ask about convergence. We will see below (the example after Lemma 8 ) that it is not always possible to find $Y$ with entries in

$$
k\{x\}:=\left\{\sum_{i=0}^{\infty} a_{i} x^{i} ; \limsup \sqrt[i]{\left|a_{i}\right|}<+\infty\right\} .
$$

Presented with such an impossibility, we then want to understand how far we are from solving these equations with convergent functions (in $k\{x\}$ ). At this point it becomes convenient to introduce an object controlling germs of stratified equations, the local fundamental group scheme $\Pi^{\text {loc }}$ (Definition 9) and study its monodromy groups, which equal algebraic quotients of $\Pi^{\text {loc }}$.

Our main result (Theorem 14) is to characterize these monodromy groups. Both the proof and the statement were inspired by Matzat and van der Put's solution of an inverse problem of differential Galois theory in positive characteristic $[\mathrm{MvdP} 03, \S 7]$. In fact, we really show the following. By twisting meticulously the construction of [MvdP03, $\S 7$ ], certain stratified differential equations over $\mathbb{A}_{k}^{1}$ (not all of them!) will keep their global monodromy groups as they come closer to the origin. Of course, there are entire families of non-trivial stratified differential equations over $\mathbb{A}_{k}^{1}$ which become trivial when approaching the origin (etale coverings and the example in $\S 4$ ). The crux of the proof is really Lemma 15 . To repeat, this is very peculiar to finite characteristic, as over $\mathbb{C}_{p}$ the monodromy will vanish when approaching the origin.

Finally, we make a topological consideration. Convergence itself can be thought of as a topological problem, i.e. find 'small neighbourhoods' in which all solutions exist as 'analytic functions' (this should be understood in the sense of Grothendieck: regular functions of the structure ring of some site). The existence of formal solutions is the question examined in the smooth topology (this is a consequence of the Artin-Néron-Popescu-Rotthaus desingularization: see [AR88] for example). This work studies convergence in the rigid topology.

Throughout, we will let $k$ be an algebraically closed field of positive characteristic $p$ complete with respect to a non-trivial non-Archimedean absolute value

$$
|\cdot|: k^{*} \rightarrow \mathbb{R}_{>0} \text {. }
$$

By a group or a group scheme we will mean an affine group scheme over $k$ (Hopf algebra).

\section{Setting}

Given a $\rho \in\left|k^{*}\right|$, let

$$
\mathscr{O}(\rho)=\left\{\sum_{i=0}^{\infty} a_{i} x^{i} \in k[[x]] ; \lim _{i}\left|a_{i}\right| \rho^{i}=0\right\}
$$

denote the affinoid algebra of analytic functions on the disc

$$
\mathbb{D}(\rho)=\{z \in k ; 0 \leqslant|z| \leqslant \rho\} .
$$

Since $\rho$ will be fixed in what follows, we will omit it from the notation and write $R:=\mathscr{O}(\rho)$ and $\mathbb{D}=\mathbb{D}(\rho)$. On $R$, there are $k$-linear homomorphisms $\partial_{n}$ defined by

$$
\partial_{n}\left(\sum_{i \geqslant 0} a_{i} x^{i}\right)=\sum_{i \geqslant n}\left(\begin{array}{c}
i \\
n
\end{array}\right) \cdot a_{i} x^{i-n}, \quad n \geqslant 0 .
$$




\section{Positive Characteristic DIFFEREnTIAL EQUATIONS}

These operators are the formal equivalent of $(1 / n !)\left(d^{n} / d x^{n}\right)$ in positive characteristic. Note that:

(i) $\partial_{0}=\mathrm{id}$;

(ii) $\partial_{n}(f g)=\sum \partial_{r}(f) \partial_{s}(g)$, where the sum is over all $r, s \geqslant 0$ with $r+s=n$;

(iii) $\partial_{m} \circ \partial_{n}=\left(\begin{array}{c}m+n \\ m\end{array}\right) \partial_{m+n}$.

The subring of $\operatorname{End}_{k}(R)$ generated over $R$ by all these operators is called the ring of differential operators and is denoted by $\mathscr{D}$.

Definition 1. Let $M$ be a finite $R$ module. A stratification on $M$ is a homomorphism of $R$-algebras

$$
\nabla: \mathscr{D} \rightarrow \operatorname{End}_{k}(M)
$$

This amounts to a family of $k$-linear homomorphisms $\left\{D_{n}\right\}_{n \in \mathbb{N}}$ satisfying:

(i) $D_{0}=\mathrm{id}$;

(ii) $D_{n}(f \cdot e)=\sum \partial_{r}(f) D_{s}(e)$ for $f \in R$ and $e \in M$, the sum being over all $r, s \geqslant 0$ with $r+s=n$;

(iii) $D_{m} \circ D_{n}=\left(\begin{array}{c}m+n \\ m\end{array}\right) D_{m+n}$.

The category of stratified modules is denoted $\operatorname{str}(\mathbb{D})$.

The reader is directed to the Appendix to Chapter 5 of [dSan06] for a more sheaf-theoretical definition of stratified modules.

The category of modules with a stratification forms an abelian tensor category as one can see by defining the tensor product stratification module

$$
\left(M, D_{n}^{M}\right) \otimes\left(N, D_{n}^{N}\right):=\left(M \otimes_{R} N, D_{n}^{M \otimes N}\right)
$$

where

$$
D_{n}^{M \otimes N}(s \otimes t)=\sum D_{i}^{M}(s) \otimes D_{j}^{N}(t),
$$

sum over all $i, j \geqslant 0$ such that $i+j=n$.

Modules with stratification are locally free. This can be done by copying the proof of Proposition 8.9 in [Kat70] (which also shows the existence of formal solutions) or using the structure of $F$-divided module (see below). In particular, $\mathbf{s t r}(\mathbb{D})$ is neutral Tannakian, if one takes the fibre at 0 as fibre functor [DM82, Definition 2.19, p. 138].

Another basic result on stratified modules is Katz's theorem. It connects the definition of stratified module to that of $F$-divided module. Let $F: R \rightarrow R$ be the absolute Frobenius.

Definition 2. The category of $F$-divided modules on $\mathbb{D}, \mathbf{F d i v}(\mathbb{D})$, has objects $\left\{M_{n}, \varphi_{n}\right\}_{n \in \mathbb{N}}$, where $M_{n}$ are finite $R$-modules and $\varphi_{n}: F^{*} M_{n+1} \rightarrow M_{n}$ is an isomorphism of $R$-modules. An arrow $\theta_{\bullet}:\left\{M_{n}, \varphi_{n}\right\} \rightarrow\left\{N_{n}, \psi_{n}\right\}$ is a sequence of $R$-linear homomorphisms $\theta_{n}: M_{n} \rightarrow N_{n}$ such that $\psi_{n} \circ F^{*}\left(\theta_{n+1}\right)=\theta_{n} \circ \varphi_{n}$.

The category $\mathbf{F d i v}(\mathbb{D})$ is obviously a tensor category; using faithful flatness of $F$, it is also abelian with kernels and cokernels defined termwise. By a lemma of Shepherd-Barron [dSan06], if $\left\{M_{i}\right\}$ is an $F$-divided module, then all the $M_{i}$ are locally free over $R$. The connection between str and Fdiv is given by the following theorem.

Theorem 3 (Katz). The categories $\mathbf{F} \operatorname{div}(\mathbb{D})$ and $\mathbf{s t r}(\mathbb{D})$ are naturally equivalent tensor categories.

See [Gie75] or Chapter 5 of [dSan06] for the construction of these equivalences. A short indication is given in $\S 3.1$ below. 


\section{J. P. P. DOs Santos}

Remark. (a) Another name for Theorem 3 is 'the theorem of Cartier-Katz' (used in [dSan06]). The presence of Cartier's name is used to remind us of the idea behind a proof.

(b) About the terminology: Grothendieck introduced stratified sheaves in [Gro68] and Gieseker introduced $F$-divided sheaves in [Gie75] (they were then called flat sheaves). In [MvdP03] stratified sheaves over a field are called ID-modules and $F$-divided sheaves have no special name. Of course, we are all talking about the same objects, but Grothendieck's terminology seems to facilitate the relation with geometry. Perhaps a more indicative name is D-module, but we have the impression that this term is overburdened.

\section{Fundamental matrices and monodromy groups}

In this section we study two aspects of stratified modules. The first $(\S 3.1)$ is the problem of finding formal solutions to a system like in (1). As remarked in the Introduction, this step goes back to Cauchy and is conceptually very useful. The notion of $F$-division plays a central role: we are able to find a basis for the solution space using a special $x$-adic limit of matrices, called a fundamental matrix.

Second (§3.2), we introduce the F-divided modules of Matzat and van der Put. The monodromy group of such a module is naturally a closed subgroup of a prescribed algebraic group - this will be important when computing the monodromy.

We keep the notation of $\S 2: \rho \in\left|k^{*}\right|, R$ is the ring of analytic functions on $\mathbb{D}=\mathbb{D}(\rho)$ and str is the category of stratified ( $F$-divided) modules over $\mathbb{D}$. Also, let $R_{n}=R \cap k\left[\left[x^{p^{n}}\right]\right]=R_{n} \subset R$ be the image of $R$ under $F^{n}: R \rightarrow R$.

\subsection{Fundamental matrices}

Stratified modules are free because they are locally free and $R$ is a principal ideal domain. Take $M$ to be a free $R$-module of rank $\mu$ with a stratification $\nabla$ and let $\left\{M=M_{0} \supset M_{1} \supset \cdots\right\}$ be the $F$-division of $M$ obtained via Katz's theorem; $M_{n}$ is the subspace of elements in $M$ killed by all $\nabla\left(\partial_{\nu}\right)$ with $0<\nu<p^{n}$. We see each $M_{n}$ as a subgroup of $M$ which is invariant under multiplication by $R_{n}$ and the natural inclusion $M_{n+1} \subset M_{n}$ induces an isomorphism $M_{n+1} \otimes_{R_{n+1}} R_{n} \rightarrow M_{n}$ of $R_{n}$-modules. Take $\mathbf{e}^{(0)}=\left(e_{1}^{(0)}, \ldots, e_{\mu}^{(0)}\right)$ as a basis of $M=M_{0}$ and let $\mathbf{e}^{(n)}$ be a basis of $M_{n}$ over $R_{n}$. If we agree to write the column vectors of $\mathbf{e}^{(n)}$ in the basis $\mathbf{e}^{(0)}$, then $\mathbf{e}^{(n)}$ is a matrix in $G L_{\mu}(R)$. Let $\varphi_{n}$ be the isomorphism $M_{n+1} \otimes_{R_{n+1}} R_{n} \rightarrow M_{n}$ and identify it with the invertible matrix with coefficients in $R_{n}$ representing the lower horizontal arrow in the diagram below.

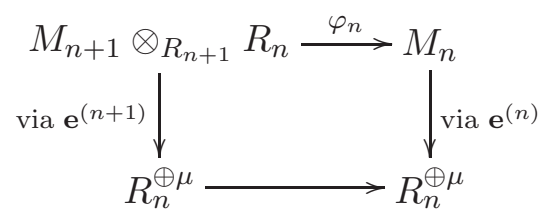

In matricial terms, we have $\mathbf{e}^{(n+1)}=\mathbf{e}^{(n)} \cdot \varphi_{n}$. If we let $f_{n}:=\varphi_{0} \cdots \varphi_{n-1}$, then the operators $D_{n}:=\nabla\left(\partial_{n}\right)$ will be given by (following the recipe given by Katz's theorem)

$$
D_{\nu}\left(\mathbf{e}^{(0)}\right):=\left(D_{\nu} e_{1}^{(0)}, \ldots, D_{\nu} e_{\mu}^{(0)}\right)=\mathbf{e}^{(0)} \cdot f_{n} \cdot \partial_{\nu}\left(f_{n}^{-1}\right), \quad 0<\nu<p^{n}
$$

If we take $\varphi_{n}(0)=\mathrm{I}$ (which is always possible if we pick $\mathbf{e}^{(n)}$ conveniently), the sequence $\left\{f_{n}\right\}$ will converge to some element $\Phi$ of $G L_{\mu}(k[[x]])$ (in the $x$-adic topology!) and the columns of $\Phi$ are 


\section{Positive Characteristic DIFFEREnTIAL EQUATIONS}

all killed by $D_{n}$ for $n>0$. To see this, it is sufficient to prove that, for any $n>0$,

$$
D_{n}\left(\mathbf{e}^{(0)} \cdot \Phi\right) \in\left(\bigcap_{m \geqslant 0} x^{m} \hat{M}\right)^{\mu}=0,
$$

where $\hat{M} \supset M$ is the $x$-adic completion of $M$. But if $\nu$ is large enough, there is a matrix $\gamma$ with power series entries such that $\Phi-f_{\nu}=x^{p^{n}} \cdot \gamma$ and hence

$$
D_{n}\left(\mathbf{e}^{(0)} \cdot \Phi\right)=D_{n}\left(\mathbf{e}^{(0)} \cdot\left(\Phi-f_{\nu}\right)\right)=x^{p^{n}} D_{n}\left(\mathbf{e}^{(0)} \cdot \gamma\right) .
$$

Also note that any $s \in \hat{M}$ which is killed by all $D_{n}$ with $n>0$ is a $k$-linear combination of the columns of $\mathbf{e}^{(0)} \cdot \Phi$ :

$$
\operatorname{Hom}_{\mathbf{s t r}(\mathbb{D}(\rho))}(\mathbb{1}, M)=\left\{\alpha \in k^{\mu} ; \Phi \cdot \alpha \in M\right\} .
$$

Definition 4. Assume all bases $\mathbf{e}^{(n)}$ have been chosen to satisfy $\varphi_{n}(0)=$ I. The matrix $\Phi$ constructed above is a fundamental matrix of $(M, \nabla)$. If all the choices are explicit, we say that $\Phi$ is the fundamental matrix.

\subsection{The modules of Matzat and van der Put}

Definition 5. Given a $k$-vector space $V$ of finite dimension and a sequence $\left\{\varphi_{n} \in G L\left(V \otimes R_{n}\right)\right\}_{n \in \mathbb{N}}$ with $\varphi_{n}(0)=\mathrm{I}$, we obtain an $F$-divided module $M\left(\varphi_{\bullet}\right)$ by setting $M\left(\varphi_{\bullet}\right)_{n}=V \otimes_{k} R_{n}$ and using the $\varphi_{n}$ as transition isomorphisms.

This association preserves the constructions of linear algebra:

$$
M\left((\varphi \otimes \psi)_{\bullet}\right)=M\left(\varphi_{\bullet}\right) \otimes M\left(\psi_{\bullet}\right), \quad M\left(\left(\varphi^{\vee}\right)_{\bullet}\right)=M\left(\varphi_{\bullet}\right)^{\vee}
$$

etc. The fundamental matrix for $M\left(\varphi_{\bullet}\right)$ is just the $x$-adic $\operatorname{limit}_{n} \lim _{n} \varphi_{0} \cdots \varphi_{n}$.

If we take $\varphi_{n} \in G\left(R_{n}\right) \subseteq \mathbb{G} \mathbb{L}\left(V \otimes R_{n}\right)$, for some algebraic subgroup $G$ of $\mathbb{G L}(V)$, then the monodromy group of $M\left(\varphi_{\bullet}\right)$ in $\operatorname{str}(\mathbb{D})$ will be naturally a subgroup of $G$ (Lemma 7 ). First we need the formal definition of monodromy or Galois group.

Definition 6. Let $(\mathfrak{A}, \otimes)$ be a neutral Tannakian category and $V$ an object. Let $\langle V\rangle_{\otimes}$ be the full subcategory of $\mathfrak{A}$ having as objects the sub-quotients (= quotients of sub-objects) of objects of the form

$$
V_{\left(b_{1}, \ldots, b_{s}\right)}^{\left(a_{1}, \ldots, a_{s}\right)}:=V_{b_{1}}^{a_{1}} \oplus \cdots \oplus V_{b_{s}}^{a_{s}}, \quad V_{b}^{a}:=V^{\otimes a} \otimes\left(V^{\vee}\right)^{\otimes b}
$$

where $s$ runs over the non-negative integers and $\left(a_{1}, \ldots, a_{s}\right),\left(b_{1}, \ldots, b_{s}\right)$ run over all the $s$-uples of non-negative integers. The monodromy group of $V$ is the group scheme associated, via Tannakian duality $[\mathrm{DM} 82,2.11]$, to the category $\langle V\rangle_{\otimes}$. The notation is: $G_{\text {mono }}(V, \mathfrak{A})$ or $G(V, \mathfrak{A})$.

Returning to the monodromy group of $M\left(\varphi_{\bullet}\right)$, note that to any representation

$$
\theta: G \rightarrow \mathbb{G} \mathbb{L}(W)
$$

we can associate another stratified module using $\theta\left(\varphi_{n}\right) \in G L\left(W \otimes R_{n}\right)$; this defines a tensor functor $\tau: \operatorname{Rep}_{k}(G) \rightarrow \operatorname{str}(\mathbb{D})$. Since every representation of $G$ is a sub-quotient of some

$$
V_{\left(b_{1}, \ldots, b_{s}\right)}^{\left(a_{1}, \ldots, a_{s}\right)}
$$

$\tau$ takes values in $\left\langle M\left(\varphi_{\bullet}\right)\right\rangle_{\otimes}$, which is equivalent to $\operatorname{Rep}_{k}\left(G_{\text {mono }}\left(M\left(\varphi_{\bullet}\right)\right.\right.$, str $\left.)\right)$. Because $\tau(V)=$ $M\left(\varphi_{\bullet}\right)$, the group homomorphism $G_{\text {mono }}\left(M\left(\varphi_{\bullet}\right)\right.$, str $) \rightarrow G$ obtained from $\tau$ is a closed embedding [DM82, 2.21, p. 139]. This shows the next result.

Lemma 7 (compare [MvdP03, Proposition 5.3]). If the matrices $\varphi_{n}$ defining the stratified module $M\left(\varphi_{\bullet}\right)$ belong to $G\left(R_{n}\right), G \subseteq \mathbb{G L}(V)$ a closed subgroup, then $G_{\text {mono }}\left(M\left(\varphi_{\bullet}\right)\right.$, str $)$ is naturally a 


\section{J. P. P. DOs SAntos}

closed subgroup of $G$. More precisely, the tensor functor $\tau: \operatorname{Rep}_{k}(G) \rightarrow \operatorname{str}$ defined by $\tau(V)=$ $M\left(\varphi_{\bullet}\right)$ induces a closed embedding.

Remark. Kedlaya proves [Ked04] that every locally free module over a polydisc is free (the analogue of the Quillen-Suslin theorem). So, one can control stratified modules over such affinoids by the method of fundamental matrices.

\section{One-dimensional modules}

Keep $\rho \in\left|k^{*}\right|$. Let $\mathbb{D}$ again denote the $\operatorname{disc}\{z \in k: 0 \leqslant|z| \leqslant \rho\}$ and $R$ its ring of analytic functions. For $M \in \operatorname{str}(D)$, let $\Phi$ denote the fundamental matrix (after a choice of the $\mathbf{e}^{(n)}$ ). Assume that $M$ is of rank one and write $\varphi_{n}=1-x^{p^{n}} \gamma_{n}$.

Lemma 8. If $r \in\left|k^{*}\right|$ is strictly smaller than $\rho$, then $M$ restricted to $\mathbb{D}(r)$ is trivial (that is, the convergence radius of $\Phi$ is $\rho$ ).

Proof. Let $r<\rho$ be in $\left|k^{*}\right|$ and let $\|\cdot\|_{r}$ denote the spectral norm of the disc $\mathbb{D}(r)$ :

$$
\left\|\sum_{i \geqslant 0} a_{i} x^{i}\right\|_{r}=\sup _{i}\left|a_{i}\right| r^{i}=\sup _{z \in \mathbb{D}(r)}\left|\sum_{i \geqslant 0} a_{i} z^{i}\right| .
$$

We have the estimates:

(i) $\left\|\varphi_{n}\right\|_{r}=1$;

(ii) $\lim _{n}\left\|\varphi_{n}-1\right\|_{r}=0$.

The lemma is a consequence of (i) and (ii) since $\|\cdot\|_{r}$ is multiplicative on $\mathscr{O}(r)$ and this algebra is complete with respect to it [BGR84, $\S 6.1 .5$, p. 234]. Both (i) and (ii) will be a consequence of Proposition 5.1.3/1 on p. 193 of [BGR84]; this proposition states that $f \in \mathscr{O}(1)-\{0\}$ is invertible if and only if $\|f\|_{1}=|f(0)|$ and $\|f-f(0)\|_{1}<\|f\|_{1}$. Because $\rho \in\left|k^{*}\right|, \mathscr{O}(\rho)$ is isometrically isomorphic to $\mathscr{O}(1)$; it follows that

$$
\left\|x^{p^{n}} \gamma_{n}\right\|_{\rho}<1 .
$$

Now (i) follows from the fact that $\left\|x^{p^{n}} \gamma_{n}\right\|_{r} \leqslant\left\|x^{p^{n}} \gamma_{n}\right\|_{\rho}<1$ and $\|\cdot\|_{r}$ is non-Archimedean. Item (ii) follows from

$$
\left\|\varphi_{n}-1\right\|_{r}=\left\|x^{p^{n}}\right\|_{r} \cdot\left\|\gamma_{n}\right\|_{r} \leqslant r^{p^{n}} \cdot\left\|\gamma_{n}\right\|_{\rho}<r^{p^{n}} \rho^{p^{-n}}=(r / \rho)^{p^{n}} .
$$

Example. If we try to adapt the same proof to the case of higher rank, we will find an obstruction in the existence of nilpotents in the algebra $\operatorname{End}_{k}\left(R^{\oplus m}\right)$ - this fact destroys the multiplicativity of norms. The entries of an invertible matrix in $\operatorname{End}\left(R^{\oplus m}\right)$ might have large spectral norms: take

$$
\left(\begin{array}{ll}
1 & * \\
0 & 1
\end{array}\right)
$$

for example. If we pick

$$
\varphi_{n}=\left(\begin{array}{cc}
1 & a_{n} x^{p^{n}} \\
0 & 1
\end{array}\right)
$$

then

$$
\Phi=\lim _{n} \varphi_{0} \cdots \varphi_{n}=\left(\begin{array}{ll}
1 & \theta \\
0 & 1
\end{array}\right), \quad \theta=\sum_{i=0}^{\infty} a_{i} x^{p^{i}} .
$$

Choosing the $a_{i}$ conveniently, this gives an example of a stratified module over $\mathbb{D}$ which, even if we shrink $\mathbb{D}$, is not trivial. On the other hand, taking $a_{i}=1$ gives a stratified module over $\mathbb{A}_{k}^{1}$ which has 'global' monodromy $\mathbb{G}_{a}$ but becomes trivial in any $\operatorname{disc} \mathbb{D}(r)$ with $r<1$. 


\section{Positive CHARACTERISTIC DIFFerential EQUATIONS}

The above stratified module has one solution (horizontal vector) $(1,0)^{\mathrm{t}}$. This vector is also fixed by all $\varphi_{n}(c)$ where $c$ runs over $k$. To produce stratified modules with no solutions at all (even in a small disc), we will avoid the existence of such a fixed vector. See $\S 6.1$.

\section{Local monodromy groups}

As we are really interested in convergence in some neighbourhood of the origin, $\operatorname{str}(\mathbb{D}(\rho))$ is not what we want. Neither is $\operatorname{str}(k\{x\})$ (see the remark below). The useful concept is contained in the next definition.

Definition 9. We define $\mathscr{T}$ to be the Tannakian category

$$
\underset{\rho}{\lim } \operatorname{str}(\mathbb{D}(\rho)), \quad \rho \in\left|k^{*}\right| .
$$

That is, the class of objects $\mathrm{Ob} \mathscr{T}$ is just the union of the class of objects in $\operatorname{str}(\mathbb{D}(\rho))$ for all $\rho \in\left|k^{*}\right|$ and the arrows between $M \in \operatorname{str}\left(\mathbb{D}\left(\rho_{1}\right)\right)$ and $N \in \operatorname{str}\left(\mathbb{D}\left(\rho_{2}\right)\right)$ are

$$
\lim _{\longrightarrow} \operatorname{Hom}_{\mathbf{s t r}(\mathbb{D}(r))}(M|\mathbb{D}(r), N| \mathbb{D}(r)), \quad r<\min \left(\rho_{1}, \rho_{2}\right) .
$$

Then $\Pi^{\text {loc }}$ is the fundamental group scheme associated to it via the fibre functor $0^{*}: \mathscr{T} \rightarrow$ $(k$-mod) (see [DM82, Theorem 2.11]).

Remark. The category $\mathscr{T}$ is not the category of stratified modules over $k\{x\}$ because the matrices defining $D_{n}$, for an object of $\mathscr{T}$, will have a common convergence radius. It is possible to construct stratified modules over $k\{x\}$ whose monodromy group is $\mathbb{G}_{m}$.

We are interested in the algebraic quotients of $\Pi^{\mathbf{l o c}}$, which are the monodromy groups of its objects. The reader should notice that the monodromy group $G(?, \mathscr{T})$ (Definition 6) is the obstruction to finding a fundamental matrix which converges in a neighbourhood of the origin: $G(?, \mathscr{T})$ is trivial if and only if such a matrix exists.

We give an adaptation of Lemma 7 to this situation.

LEMma 10. (i) We use the same notation as in Lemma 7. The monodromy group of $M\left(\varphi_{\bullet}\right)$ in the category $\mathscr{T}$ is a closed subgroup of the monodromy group of $M\left(\varphi_{\bullet}\right)$ in $\operatorname{str}(\mathbb{D}(\rho))$. In particular, it is a closed subgroup of $G$.

(ii) Moreover, we let $\rho^{\prime}<\rho$ be in $\left|k^{*}\right|$ and let $M$ (respectively $\left.M^{\prime}\right)$ denote an object of $\operatorname{str}(\mathbb{D}(\rho))$ (respectively its restriction to $\mathbb{D}\left(\rho^{\prime}\right)$ ). Then there exists a natural closed embedding

$$
\iota: G\left(M^{\prime}, \operatorname{str}\left(\mathbb{D}\left(\rho^{\prime}\right)\right)\right) \longrightarrow G(M, \operatorname{str}(\mathbb{D}(\rho)))
$$

of the monodromy groups and under this homomorphism the representation corresponding to $M$ restricts to the representation corresponding to $M^{\prime}$.

Proof. The monodromy group of $M\left(\varphi_{\bullet}\right)$ in $\operatorname{str}(\mathbb{D}(\rho))$ is the Tannakian fundamental group associated to the category of all sub-quotients in $\operatorname{str}(\mathbb{D}(\rho))$ of $M\left(\varphi_{\bullet}\right)_{\left(b, \ldots, b_{s}\right)}^{\left(a_{1}, \ldots, a_{s}\right)}$ (notation is that of (3)). The monodromy of $M\left(\varphi_{\bullet}\right)$ in $\mathscr{T}$ is analogous and the lemma is just an application of [DM82, Proposition 2.21, p. 139].

The second part is just as easy (and uses again the same proposition in [DM82]).

Corollary 11. Let $M$ be an object of $\mathscr{T}$. Then there are a $\rho \in\left|k^{*}\right|$ and an $M_{\rho}$ such that $M$ is the restriction of $M_{\rho}$ and the natural homomorphism $G(M, \mathscr{T}) \rightarrow G\left(M_{\rho}, \operatorname{str}(\mathbb{D}(\rho))\right)$ is an isomorphism.

Proof. This is a consequence of the second part of Lemma 10 and the fact that all monodromy groups in $\operatorname{str}(\mathbb{D}(r))$ are reduced (see $[\mathrm{dSan} 06, \mathrm{ch} .1])$. 


\section{J. P. P. DOs SAntos}

Given a reduced algebraic group $G$ over $k$, we introduce the groups $p(G)$ and $G^{(p)}$. The group $p(G)$ is the smallest closed subgroup of $G$ containing all the elements of order a power of $p$. Obviously it is a normal subgroup.

Lemma 12 [MvdP03, Claim, p. 28]. The notation is as above. We have that:

(i) $p(G)$ is an algebraic subgroup of $G$;

(ii) the connected component of the quotient $G^{(p)}:=G / p(G)$ is either trivial or a torus and $\pi_{0} G^{(p)}$ is a finite group of order prime to $p$.

Lemma 13. Let $G$ be an algebraic quotient of $\Pi^{\text {loc }}$. Then we have that:

(i) $G$ is reduced;

(ii) if $G$ is finite, it is trivial;

(iii) $G$ is connected and equals $p(G)$.

Proof. The proof of (i) follows from Corollary 11 and the fact (which was used to prove the corollary) that all the monodromy groups of $\operatorname{str}(\mathbb{D}(\rho))$ are reduced. The validity of (ii) is a consequence of $(i)$ and the fact that the local ring $\lim _{\rightarrow \rho} \mathscr{O}(\mathbb{D}(\rho))=k\{x\}$ is strict henselian [Nag62, Theorem 45.5, p. 193] - for more information on $F$-divided modules and etale coverings the reader is directed to [dSan06, ch. 1]. We now prove (iii). By item (ii), $\Pi^{\text {loc }}$ has no non-trivial finite etale quotients, so $\pi_{0}\left(G^{(p)}\right)=\{1\}$ and $G^{(p)}=\left(G^{(p)}\right)^{\circ}$, which should be either trivial or a torus. By Lemma 8, every diagonal quotient of $\Pi^{\text {loc }}$ is trivial. Hence, $G^{(p)}=\{1\}$.

This lemma takes care of the 'only if' part of our following main result.

Theorem 14. An algebraic group $G$ is a quotient of $\Pi^{\text {loc }}$ if and only if it is reduced, connected and generated, as an algebraic group, by its elements of order a power of $p$.

The proof is given below in Theorem 19: given a connected reduced algebraic group $G=p(G)$, we construct a module in $\mathscr{T}$ whose monodromy is $G$.

\section{Differential equations with no non-trivial convergent solutions and proof of Theorem 14}

In this section we will give a proof of Theorem 14 (our main theorem). The idea is as follows. Let $G=p(G) \subseteq \mathbb{G L}(m)$. We want to find matrices $\varphi_{n} \in G\left(R_{n}\right)$ such that the canonical embedding of the monodromy group $H$ of $M\left(\varphi_{\bullet}\right)$ in $G$ (Lemma 10) is in fact an isomorphism. Chevalley taught us how to deal with the construction of quotients $G / H$ by finding lines in some representation $W$ of $G$ which are fixed by $H$ but not by $G$ (by general Tannakian theory, such a representation is related to the standard representation $G \subseteq \mathbb{G} \mathbb{L}(m)$ ). We follow this idea with the constant support of Lemma 8, which states that such a line will in fact correspond to a fixed element for the $H$-action (convergent solution). The proof of Theorem 14 will follow from the non-existence of such fixed vectors (if we chose the $\varphi_{n}$ carefully!). Section 6.1 shows what sort of constraint will appear if there exists a convergent solution and $\S 6.2$ shows how to pick the matrices $\varphi_{n}$ so as to violate these constraints.

\subsection{Some properties impeding convergent solutions}

In this section we study conditions on the matrices $\varphi_{n}$ so that $M=M\left(\varphi_{\bullet}\right)$ has no convergent solution (Lemma 15) Convergent means that there exists a horizontal morphism from the trivial object of $\operatorname{str}(\mathbb{D}(\rho))$ to $M$, for some small $\rho$. Of course, this is equivalent to finding an $\alpha \in V=0^{*} M$ 


\section{Positive CHARACTERISTIC DIFFERENTIAL EQUATIONS}

with $\Phi \cdot \alpha \in V \otimes \mathscr{O}(\rho)$, as any element of $\hat{M}=M \otimes k[[x]]$ killed by all differential operators of positive order is a $k$-linear combination of the columns of $\Phi$.

In order to see what kind of constraint the convergence of $\Phi \cdot \alpha$ will impose on the norms $\left\|\varphi_{n} \cdot \alpha\right\|_{\rho}$, we want, first, to take $\varphi_{n}$ to be polynomial. This will allow us to use the degree to compare the coefficients of $\Phi \cdot \alpha$ with those of $\varphi_{n} \cdot \alpha$.

We would like the degrees to grow fast so that $\varphi_{0} \cdots \varphi_{n+1}$ is the sum of $\varphi_{0} \cdots \varphi_{n}$ with terms of higher degree. As this is not really feasible (for the pair $n, n+1$ ), we follow [MvdP03] and introduce very large 'gaps' in the sequence $\varphi_{n}$ :

$$
\varphi_{0} \cdots \varphi_{n}=\varphi_{0} \cdots \varphi_{n+1}=\cdots=\varphi_{0} \cdots \varphi_{n^{\prime}-1}
$$

for some $n^{\prime} \gg n$ (see Property 2 below).

Finally, in order to avoid the situation in which the degree of $\varphi_{0} \cdots \varphi_{n} \alpha$ stays bounded, we will impose that the group generated by the various $\varphi_{n}(k)$ with $n \geqslant N$ generate a subgroup of $\mathbb{G L}(V)$ which does not fix $\alpha$ (Property 4).

Let $A_{n}:=k\left[x^{p^{n}}\right]$ and let $V$ be a $k$-vector space with a fixed basis: $k^{m} \cong V$. Take matrices $\varphi_{n} \in G L\left(V \otimes_{k} A_{n}\right)$ with the following properties.

Property 1. One has $\varphi_{n}(0)=\mathrm{I}$.

Property 2. There is an increasing sequence of positive integers $\left\{n_{i}\right\}_{i \in \mathbb{N}}$ such that $\varphi_{n}=\mathrm{I}$ if $n \notin\left\{n_{i}\right\}$. Also, $\left\{n_{i+1}-n_{i}\right\}_{i \in \mathbb{N}}$ tends to infinity.

Property 3. If we write $\varphi_{n}=\mathrm{I}+\Gamma_{n}$, then the degree of $\Gamma_{n}$ in $x$ is bounded by $b p^{n}, b>0$.

Property 4. Let $G$ be the subgroup of $\mathbb{G} \mathbb{L}(V)$ generated by the subset $\bigcup_{n \geqslant 0} \varphi_{n}(k)$. Then, for any $N \in \mathbb{N}, G$ is also generated by $\bigcup_{n \geqslant N} \varphi_{n}(k)$.

We note that the degree of a matrix in $G L\left(V \otimes_{k} k[x]\right)=G L_{m}(k[x])$ is well defined as is the degree of a vector $f \in V \otimes_{k} k[x]$. Also, given an element

$$
\xi=\sum_{i=0}^{\infty} v_{i} \otimes x^{i} \in V \otimes_{k} k[[x]],
$$

we define, for some $\rho \in\left|k^{*}\right|$,

$$
\|\xi\|_{\rho}=\sup _{i}\left\{\left|v_{i}\right| \rho^{i}\right\}
$$

where $|\cdot|: V \rightarrow \mathbb{R}_{\geqslant 0}$ is the maximum norm with respect to the basis giving $k^{m} \cong V$. Under these conventions, $V \otimes_{k} \mathscr{O}(\rho)$ is the subspace of all $\xi$ with $\lim _{i}\left|v_{i}\right| \rho^{i}=0$.

Note that picking a different basis for $V$ gives a different $\|\cdot\|_{\rho}: V \otimes_{k} \mathscr{O}(\rho) \rightarrow \mathbb{R}_{\geqslant 0}$, but the topology is the same. In particular, the concept of a sequence in $V \otimes_{k} \mathscr{O}(\rho)$ having bounded norm is well defined.

We will also find it convenient to call the monomials $v_{i} \otimes x^{i}$ of $\xi$ in (4) the terms of $\xi$.

The matrices $\varphi_{n}$ define a stratified module $M\left(\varphi_{\bullet}\right)$ over $\mathbb{D}(\rho), \rho \in\left|k^{*}\right|$, and we let $\Phi$ be the fundamental matrix (in the notation of Definition 5). Recall that we are denoting by $G$ the subgroup of $\mathbb{G} \mathbb{L}(V)(k)$ generated by $\bigcup_{n \geqslant 0} \varphi_{n}(k)$.

Lemma 15. The notation is as above. Assume that, for some $\alpha \in V-\{0\}, \Phi \cdot \alpha \in V \otimes_{k} k[[x]]$ is actually in $V \otimes_{k} \mathscr{O}(\rho)$. If $\alpha$ is not fixed by $G$ then there exists an infinite subsequence $S \subseteq\left\{n_{i}\right\}_{i \in \mathbb{N}}$ such that $\Gamma_{s} \cdot \alpha \neq 0$ and $\left\|\Gamma_{s} \cdot \alpha\right\|_{\rho}$ is bounded for all $s \in S$.

Proof. First of all, $\Phi \cdot \alpha \in V \otimes_{k} \mathscr{O}(\rho)$ if and only if $\varphi_{n}^{-1} \cdots \varphi_{0}^{-1} \Phi \cdot \alpha \in V \otimes_{k} \mathscr{O}(\rho)$. Thus, we can assume that $p^{n_{i+1}}>b p^{n_{i}}$ for all $i \geqslant 0$. Let $\Phi_{n}$ denote $\varphi_{0} \cdots \varphi_{n}$. It is easy to see that the degree 


\section{J. P. P. DOs Santos}

of $\Phi_{n_{i}} \cdot \alpha$ is less than $p^{n_{i+1}}$. These normalizations are made to study the terms of $\Phi \cdot \alpha$ and to show that they are related to the $\Gamma_{*} \cdot \alpha$ in a way that the condition $\Phi \cdot \alpha \in V \otimes_{k} \mathscr{O}(\rho)$ gives the desired bound.

More precisely, take an arbitrary $i \in \mathbb{N}$ and let us study the next step $\Phi_{n_{i+1}} \cdot \alpha$ in the sequence. We have

$$
\begin{aligned}
\Phi_{n_{i+1}} \cdot \alpha & =\Phi_{n_{i}} \cdot \alpha+\left(\Phi_{n_{i+1}}-\Phi_{n_{i}}\right) \cdot \alpha \\
& =\Phi_{n_{i}} \cdot \alpha+\Phi_{n_{i}} \cdot \Gamma_{n_{i+1}} \cdot \alpha .
\end{aligned}
$$

Of course, we might have $\varphi_{n_{i+1}} \cdot \alpha=\alpha$ and the second term above is zero. But, since $\alpha \notin V^{G}$, Property 4 above guarantees that there exists $j>i$ such that $\varphi_{n_{\nu}} \cdot \alpha=\alpha$ for all $\nu \in\{i+1, \ldots, j-1\}$, but $\Gamma_{n_{j}} \cdot \alpha \neq 0$. Hence $\Phi_{n_{i}} \cdot \Gamma_{n_{j}} \cdot \alpha \neq 0$ ( $\Phi_{n}$ is invertible) and

$$
\Phi_{n_{j}} \cdot \alpha=\Phi_{n_{i}} \cdot \alpha+\Phi_{n_{j-1}} \cdot \Gamma_{n_{j}} \cdot \alpha \text { and } \Phi_{n_{j-1}} \cdot \Gamma_{n_{j}} \cdot \alpha \neq 0 .
$$

Now, the condition on the degrees shows that the degree of $\Phi_{n_{i}} \cdot \alpha$ is less than the term of least degree in $\Phi_{n_{j-1}} \cdot \Gamma_{n_{j}} \cdot \alpha$ since the degree of $\Phi_{n_{i}} \cdot \alpha$ is at most $b p^{n_{i}}$ and non-zero terms of $\Gamma_{n_{j}} \cdot \alpha \neq 0$ have degree greater than or equal to $p^{n_{j}}$. This has the important consequence that all terms ? $\otimes x^{d}$ appearing in $\Phi_{n_{i}} \cdot \alpha$ also appear in $\Phi \cdot \alpha$. If we follow the same reasoning with $j$ in place of $i$, it follows that the same terms ? $\otimes x^{d}$ appearing in $\Phi_{n_{j-1}} \cdot \Gamma_{n_{j}} \cdot \alpha$ appear in $\Phi \cdot \alpha$. Because $\Phi \cdot \alpha$ belongs to $V \otimes_{k} \mathscr{O}(\rho)$, there exists a constant $c>0$ such that $\left\|\Phi_{n_{j-1}} \cdot \Gamma_{n_{j}} \cdot \alpha\right\|_{\rho} \leqslant c$.

All terms in $\Phi_{n_{j-1}} \cdot \Gamma_{n_{j}} \cdot \alpha$ are of the form ? $\otimes x^{d}$, with

$$
d=\varepsilon_{0} r_{0} p^{n_{0}}+\cdots+\varepsilon_{j-1} r_{j-1} p^{n_{j-1}}+r_{j} p^{n_{j}}, \quad \varepsilon \in\{0,1\}, r \in\{1, \ldots, b\} .
$$

By the (easy) Lemma 16 below, the terms of $\Phi_{n_{j-1}} \Gamma_{n_{j}} \cdot \alpha$ whose degree is between $p^{n_{j}}$ and $b p^{n_{j}}$ will be the terms of corresponding degree in $\Gamma_{n_{j}} \cdot \alpha$. Hence, $\left\|\Gamma_{n_{j}} \cdot \alpha\right\|_{\rho} \leqslant c$, as we wanted.

LEMma 16. Let $\{0, \ldots, b\}^{\oplus \mathbb{N}}$ be the restricted product of the set $\{0, \ldots, b\}$ with respect to the subset $\{0\}$. Assume that $p^{n_{i+1}}>b p^{n_{i}}$ for all $i \geqslant 0$. Then the map $\{0, \ldots, b\}^{\oplus \mathbb{N}} \rightarrow \mathbb{N}$ given by

$$
\left(m_{0}, m_{1}, \ldots\right) \mapsto \sum_{i=0}^{\infty} m_{i} p^{n_{i}}
$$

is injective.

Proof. The proof is very easy.

\subsection{Proof of Theorem 14}

Take any reduced connected algebraic group $G=p(G)$. Note that any unipotent subgroup of $G$ is contained in $p(G)$, and hence the closed normal subgroup $\mathbb{U}(G)$ generated by all the unipotent and connected closed subgroups of $G$ is contained in $p(G)$. The quotient $G / \mathbb{U}(G)$ is a torus or trivial and hence contains no elements of order a power of $p$; it follows that $\mathbb{U}(G)=p(G)$. Using this we have the following result.

Lemma 17 [MvdP03, 7.6]. Let $G$ equal $p(G)$ as above. Then there are morphisms $u_{1}, \ldots, u_{h}: \mathbb{G}_{a} \rightarrow$ $G$ taking 0 to the identity such that $G$ is generated by $\bigcup_{i} u_{i}(k)$.

Now, let $u_{1}, \ldots, u_{h}$ be as in Lemma 17 above. Choose a sequence $\left\{a_{n}\right\}_{n=1}^{\infty}$ in $k^{*}$ such that $\left|a_{n}\right|$ grows very fast:

$$
\lim _{n \rightarrow+\infty} \frac{\log \left|a_{n}\right|}{p^{n}}=+\infty
$$

In particular the inequality

$$
\left|a_{n}\right| \leqslant c r^{p^{n}}, \quad c, r>0
$$

is only possible for finitely many $n$. 


\section{Positive Characteristic DIFFEREnTIAL EQUATIONS}

Pick a sequence of non-negative integers $\left\{n_{i}\right\}_{i \in \mathbb{N}}$ as in Property 3 and define elements $\varphi_{n} \in$ $G\left(A_{n}\right)$ by

$$
\varphi_{n}:= \begin{cases}\mathrm{I}, & \text { if } n \notin\left\{n_{i}\right\}_{i \in \mathbb{N}}, \\ u_{l}\left(a_{n_{i}} x^{p^{n_{i}}}\right), & \text { if } n=n_{i} \text { and } i \equiv l \bmod h .\end{cases}
$$

Let $\pi: G \rightarrow \mathbb{G} \mathbb{L}(V)$ be a representation. Writing

$$
\pi \circ u_{l}(x)=\mathrm{I}+\sum_{r=1}^{b} \gamma_{l r}^{(\pi)} \otimes x^{r}, \quad \gamma_{l r}^{(\pi)} \in \operatorname{End}_{k}(V),
$$

it follows that

$$
\pi \varphi_{n_{i}}=\mathrm{I}+\sum_{r=1}^{b} a_{n_{i}}^{r} \cdot \gamma_{l r}^{(\pi)} \otimes x^{r p^{n_{i}}}, \quad \text { if } i \equiv l \bmod h .
$$

So, if $\alpha \in V$ is such that

$$
\left\|\pi \varphi_{n_{i}} \cdot \alpha-\alpha\right\|_{\rho} \leqslant c, \quad \text { for some } c>0
$$

we have for $i \equiv l \bmod h$

$$
\left|\gamma_{l r}^{(\pi)} \cdot \alpha\right| \cdot\left|a_{n_{i}}\right|^{r} \leqslant c \rho^{-r p^{n_{i}}}
$$

In the presence of growth condition (5), inequality (9) is possible for infinitely many values of $i$ if and only if $\gamma_{l r}^{(\pi)} \cdot \alpha=0$.

Let $\Phi$ be the fundamental matrix for the module $M\left(\pi \varphi_{\bullet}\right)$. If, for some $\alpha \in V-\{0\}, \Phi \cdot \alpha$ actually belongs to $V \otimes_{k} \mathscr{O}(\rho)(\rho>0)$, by Lemma 15, $\alpha$ is fixed by $G$. In a nutshell, we have the following lemma.

Lemma 18. Let $G=p(G)$ and let $u_{1}, \ldots, u_{h}: \mathbb{G}_{a} \rightarrow G$ be morphisms as in Lemma 17. Let $\left\{a_{n}\right\}_{n \in \mathbb{N}}$ be a sequence in $k^{*}$ satisfying (5) and let $\left\{n_{i}\right\}_{i \in \mathbb{N}}$ be a sequence of integers as in Property 2 above. Take $\left\{\varphi_{n}\right\}_{n \in \mathbb{N}}$ as in (7) and, for some representation $\pi: G \rightarrow \mathbb{G} \mathbb{L}(V)$, let $\Phi$ be the fundamental matrix for $M\left(\pi \varphi_{\bullet}\right)$. If $\alpha \in V$ is such that $\Phi \cdot \alpha \in V \otimes_{k} \mathscr{O}(\rho)$, then $\alpha \in V^{G}$.

Theorem 19. Keep the notation of Lemma 18 and assume that $\pi$ embeds $G$ as a closed subgroup of $\mathbb{G L}(V)$. Then the canonical inclusion of the monodromy group $G_{\text {mono }}:=G\left(M\left(\pi \varphi_{\bullet}\right), \mathscr{T}\right) \subseteq G$ given in Lemma 10 is in fact an equality.

Proof. Assume that $G_{\text {mono }} \neq G$ and use Lemma 21 below to obtain a representation $\theta: G \rightarrow \mathbb{G L}(W)$ with

$$
\theta=\wedge^{r}\left(\pi_{b_{1}}^{a_{1}} \oplus \cdots \oplus \pi_{b_{s}}^{a_{s}}\right)
$$

such that $G_{\text {mono }}$ fixes a line $L \subset W$ which is not fixed by $G$. Since $G_{\text {mono }}$ acts trivially on $L$ (by Lemma 8), there exists $\alpha \in W^{G_{\text {mono }}}$ which is not in $W^{G}$. Now, the representation of $\Pi^{\text {loc }}$ obtained by the composition

$$
\Pi^{\text {loc }} \rightarrow G_{\text {mono }} \rightarrow G \rightarrow \mathbb{G L}(W)
$$

induces, by Tannakian duality, an $N \in \mathscr{T}$ which is just $M\left(\theta\left(\varphi_{\bullet}\right)\right)$, since

$$
\bigwedge^{r}\left(M\left(\pi \varphi_{\bullet}\right)_{\left(b_{1}, \ldots, b_{s}\right)}^{\left(a_{1}, \ldots, a_{s}\right)}\right) \cong M\left(\bigwedge^{r}\left(\left(\pi \varphi_{\bullet}\right)_{\left(b_{1}, \ldots, b_{s}\right)}^{\left(a_{1}, \ldots, a_{s}\right)}\right)\right) .
$$

Let $\Psi$ be the fundamental matrix of the stratified module $M\left(\theta \varphi_{\bullet}\right)$. By Lemma 20 below, it follows that $\Psi \cdot \alpha$ is in $W \otimes_{k} \mathscr{O}(\rho)$ for some $\rho \in\left|k^{*}\right|$. By Lemma 18, $\alpha \in W^{G}$, which is a contradiction. This shows that the representation $W$ cannot exist and hence that $G_{\text {mono }}=G$. 


\section{J. P. P. DOs Santos}

Lemma 20. Let $V$ be a $k$-vector space and let $\varphi_{n} \in \mathbb{G} \mathbb{L}(V)\left(A_{n}\right)$ be such that $\varphi_{n}(0)=\mathrm{I}$. Let $\Phi$ denote the fundamental matrix for $M\left(\varphi_{\bullet}\right)$. If $\alpha \in V$ is fixed by $\Pi^{\mathbf{l o c}}$, then $\Phi \cdot \alpha$ belongs to $V \otimes_{k} \mathscr{O}(\rho)$ for some $\rho \in\left|k^{*}\right|$.

Proof. Let $a: \mathbb{1} \rightarrow V$ be the $\Pi^{\text {loc }}$-equivariant map that takes 1 to $\alpha$. This corresponds to an arrow

$$
A: \mathbb{1} \rightarrow M\left(\varphi_{\bullet}\right)
$$

in some $\operatorname{str}(\mathbb{D}(\rho))$, with $\rho \in\left|k^{*}\right|$, such that taking the fibre at 0 gives $a$ back. But $A(1)$ is of the form $\Phi \cdot \beta$ for some $\beta \in V$. Since $\Phi(0)=\mathrm{I}$, we have $\alpha=(\Phi \cdot \beta)(0)=\beta$ and because $A$ is defined over $\mathbb{D}(\rho)$ we have $\Phi \cdot \alpha=\Phi \cdot \beta=A(1) \in V \otimes_{k} \mathscr{O}(\rho)$.

Lemma 21. Let $H \subseteq G \subseteq \mathbb{G} \mathbb{L}(V)$ and assume that $H \neq G$. Then there exist $r, a_{1}, \ldots, a_{s}, b_{1}, \ldots$, $b_{s} \in \mathbb{N}$ such that $H$ is the stabilizer of a line in

$$
\bigwedge^{r}\left(V_{\left(b_{1}, \ldots, b_{s}\right)}^{\left(a_{1}, \ldots, a_{s}\right)}\right)
$$

Proof. From [Wat79, Corollary 1.16, p. 122] there exists a representation $U$ of $G$ which has $H$ as the stability group of a line $L$. Such a representation $U$ is of the form $U^{\prime} / U^{\prime}$ with subrepresentations $U^{\prime} \subset U^{\prime} \subseteq V_{\left(b_{1}, \ldots, b_{s}\right)}^{\left(a_{1}, \ldots, a_{s}\right)}$. It follows easily that $H$ is the stability group of a subspace $U^{(3)} \subset V_{\left(b_{1}, \ldots, b_{s}\right)}^{\left(a_{1}, \ldots, a_{s}\right)}$. The rest is linear algebra.

Remark. If $k$ is only perfect, Lemma 7.5 of [MvdP03] still holds. As a consequence, Lemma 17 above will also hold (with the obvious changes) if we assume that the reduced and connected $k$-group scheme $G$ is generated by its closed $k$-subgroups $H \subseteq G$ where $H$ is connected, reduced and unipotent. The previous construction will then extend to this situation: if $G$ is as before, then it is an algebraic quotient of $\Pi^{\text {loc }}$. I thank the referee for calling my attention to this fact.

\section{REFERENCES}

AR88 M. Artin and C. Rotthaus, A structure theorem for power series rings, in Algebraic geometry and commutative algebra, vol. I (Kinokuniya, Tokyo, 1988), 35-44.

BGR84 S. Bosch, U. Güntzer and R. Remmert, Non-Archimedean analysis, Grundlehren der Mathematischen Wissenschaften, vol. 261 (Springer, Berlin, 1984).

Car61 H. Cartan, Théorie élémentaire des fonctions analytiques d'une ou plusieurs variables complexes (Hermann, Paris, 1961).

DM82 P. Deligne and J. Milne, Tannakian categories, Lecture Notes in Mathematics, vol. 900 (Springer, Berlin, 1982), 101-228.

DGS94 B. Dwork, G. Gerotto and F. Sullivan, An introduction to G-functions, Annals of Mathematics Studies, vol. 133 (Princeton University Press, Princeton, NJ, 1994).

Gro68 A. Grothendieck, Crystals and the de Rham cohomology of schemes, in Dix exposés sur la cohomologie des schémas, Advanced Studies in Pure Mathematics (North-Holland, Amsterdam, 1968), 306-358.

Gie75 D. Gieseker, Flat vector bundles and the fundamental group in non-zero characteristics, Ann. Sc. Norm. Super. Pisa, Cl. Sci. (4) 2 (1975), 1-31.

Kat70 N. M. Katz, Nilpotent connections and the monodromy theorem: applications of a result of Turrittin, Publ. Math. Inst. Hautes Études Sci. 39 (1970), 175-232.

Ked04 K. Kedlaya, Full faithfulness for overconvergent F-isocrystals, in Geometric aspects of Dwork theory, vol. II (Walter de Gruyter, Berlin, 2004), 819-835.

MvdP03 B. H. Matzat and M. van der Put, Iterative differential equations and the Abhyankar conjecture, J. reine angew. Math. 557 (2003), 1-52. 


\section{Positive Characteristic Differential EqUations}

Nag62 M. Nagata, Local rings, Interscience Tracts in Pure and Applied Mathematics, vol. 13 (Interscience, New York, 1962).

dSan06 J. P. P. dos Santos, Fundamental groups in algebraic geometry, PhD thesis, University of Cambridge (2006).

Spr81 T. A. Springer, Linear algebraic groups, Progress in Mathematics, vol. 9 (Birkhäuser, Boston, 1981).

Wat79 W. C. Waterhouse, Introduction to affine group schemes, Graduate Texts in Mathematics, vol. 66 (Springer, New York, 1979).

João Pedro Pinto dos Santos joao-pedro.dos-santos@univ-rennes1.fr

IRMAR, Université de Rennes 1, Campus de Beaulieu, 35042 Rennes, France 